\title{
Seletividade de Herbicidas a Cinco Tipos de Gramas ${ }^{1}$
}

\author{
Herbicide Selectivity to Five Species of Turfgrasses \\ CHRISTOFFOLETI, P.J. ${ }^{2}$ e ARANDA, A.N. ${ }^{3}$
}

\begin{abstract}
RESUMO - Dentre as opções de controle de plantas daninhas em gramados, destaca-se o uso de herbicidas, porém poucas informações existem na literatura sobre a sua seletividade a gramados. Assim, foi desenvolvida a presente pesquisa em casa de vegetação da ESALQ Piracicaba, com o objetivo de testar a seletividade de herbicidas aplicados em pré-plantio (sulfentrazone a 1,4 kg i.a. ha-1 e oxadiazon a 1,0 kg i.a. ha-1) e em pós-emergência (halosulfuron a 112,5 g i.a. ha-1, 2,4-D a 2,010 kg i.a. ha-1 e MSMA a 2,4 kg i.a. ha-1), em cinco espécies de gramas (família Poaceae): são-carlos (Axonopus affinis), esmeralda (Zoysiajaponica), bermuda (Cynodon dactylon), zoysia (Zoysia matrella) e santo-agostinho (Stenotaphrum secundatum). As conclusões gerais deste trabalho de pesquisa foram de que a seletividade de herbicidas aos diversos tipos de grama é função principalmente da espécie de grama e do tipo e da dose do herbicida aplicado. Dentre os herbicidas testados, destacam-se como mais seletivos o halosulfuron na dose de $112,5 \mathrm{~g}$ i.a. ha-1 e o 2,4-D na de 2,010 kg i.a. ha-1; as espécies de grama do gênero Zoysia (esmeralda [Z. japonica] e zoysia [Z. matrella]), de maneira geral, são mais tolerantes aos herbicidas testados nesta pesquisa.
\end{abstract}

Palavras-chave: Axonopus affinis, Zoysia japonica, Cynodon dactylon, Zoysia matrella, Stenotaphrum secundatum.

ABSTRACT - Among the weed control options, herbicide is certainly one to be considered for weed control in turfgrass; however, little has been published about herbicide selectivity, specially under Brazilian landscape conditions. Therefore, this research was conducted at USP/ESALQ, Piracicaba-SP, Brazil, to evaluate herbicide selectivity to turfgrass of pre-planting application herbicides (sulfentrazone at $1.4 \mathrm{~kg}$ a.i.ha $\mathrm{h}^{-1}$ and oxadiazon $1.0 \mathrm{~kg}$ a.i.ha ${ }^{-1}$ ) and post-emergence application herbicides (halosulfuron at $112.5 \mathrm{~g}_{\text {a.i.ha }}{ }^{-1}$, 2,4-D at $2.010 \mathrm{~kg}$ a.i.ha $\mathrm{A}^{-1}$ and MSMA at $2.4 \mathrm{~kg}$ a.i.ha $\mathrm{h}^{-1}$, in five species of turfgrass: Axonopus affinis Chase, Zoysia japonica, Cynodon dactylon, Zoysia matrella and Stenotaphrum secundatum. It was concluded that herbicide selectivity to the turfgrass species studied is a function of the grass species and herbicide type and rate sprayed. Among the tested herbicides, halosulfuron at $112.5 \mathrm{~g}$ a.i.ha-1 and 2,4-D at $2010 \mathrm{~g}$ a.i.ha $\mathrm{a}^{-1}$ were the most selective ones, and the grass species of the genus Zoysia (Z. aponica and $\mathbf{Z}$. matrella), were, in general, the most tolerant to all the herbicides tested.

Key words: Axonopus affinis, Zoysia japonica, Cynodon dactylon, Zoysia matrella, Stenotaphrum secundatum.

\section{INTRODUÇÃO}

Os gramados constituem há séculos um dos componentes paisagísticos mais importantes.
Muitas das espécies de grama utilizadas atualmente foram desenvolvidas a partir de pradarias e pastagens. No entanto, os gramados implantados na atualidade, com espécies e

Recebido para publicação em 24/1/2001 e na forma revisada em 2/7/2001.

2 Professor Associado da Universidade de São Paulo, Escola Superior de Agricultura "Luiz de Queiroz", Departamento de Produção Vegetal - Caixa Postal 09, 13418-900 Piracicaba-SP, <pjchrist@ carpa.ciagri.usp.br> - tel.: 194294190 / fax 19429 4385. ${ }^{3}$ M.S., Professora da Universidade Nacional de Assuncion - UNA, Paraguai; Pós-Graduanda em Agronomia, Universidade de São Paulo, Escola Superior de Agricultura "Luiz de Queiroz", Área de Concentração Fitotecnia. 
híbridos de grama de grande beleza, estão a uma longa distância de seus antecessores, necessitando, portanto, de cuidados especiais em sua manutenção. Dentre estes cuidados destaca-se a necessidade de aparas constantes da grama, fertilização, aeração do solo, irrigação e controle de plantas daninhas (Itograss, 1997).

Um gramado bem aparado é certeza de sucesso em um projeto paisagístico, porém o uso de cortadores para esta operação é trabalhoso e caro; assim, têm sido testados herbicidas com o objetivo de inibir o crescimento da grama-batatais (Paspalum notatum) e, dessa forma, evitar a operação de roçagem da grama (Freitas et al., 1997). Entretanto, o herbicida deve inibir o crescimento da grama sem afetar sua beleza e coloração verde característica. $\mathrm{O}$ herbicida cimetacarb apresenta bom potencial para ser utilizado como regulador de crescimento de gramados, sem alterar a coloração da grama, evitando podas até 90 dias após sua aplicação, a partir da dose de $0,25 \mathrm{~kg}$ i.a ha-1 (Freitas et al., 1997). Da mesma forma, esses autores concluíram que a aplicação do herbicida clethodim nas doses de 0,036 e $0,048 \mathrm{~kg}$ i.a ha-1 causou sintomas visuais de toxicidade, deixando o gramado com coloração púrpura; no entanto, após 15 a 20 dias da aplicação do herbicida, iniciou-se um processo de recuperação das injúrias sofridas pela planta, até total recuperação dos efeitos fitotóxicos do herbicida.

O controle de plantas daninhas por meio de herbicidas depende da seleção de herbicidas eficientes no controle, porém sem causar injúrias indesejáveis ao gramado. Na literatura existem trabalhos que procuram estudar a seletividade de herbicidas a gramados. Bhowmik e Neal (1995) descreveram duas estratégias de controle de plantas daninhas em gramados utilizando herbicidas, sendo a primeira relacionada com o uso de herbicidas em pré-emergência para controle de gramíneas e folhas largas e, a segunda, com a aplicação seqüencial de herbicidas em pré-emergência, seguida pela aplicação em pré ou pós-emergência, dependendo do tipo de planta daninha presente, da utilização do gramado e do local, para o controle de gramíneas e folhas largas.

As pesquisas no Brasil sobre seletividade de herbicidas a gramados são ainda muito escassas, apesar de alguns trabalhos terem sido apresentados em congressos (Carmona \& Silva, 1997; Freitas et al. 1997; Foloni \& Camposilvam, 1997). Dessa forma, foi desenvolvido o presente estudo com o objetivo de testar a tolerância das gramas são-carlos (Axonopus affinis), esmeralda (Zoysiajaponica), bermuda (Cynodon dactylon), zoysia (Zoysia matrella) e santo-agostinho (Stenotaphrum secundatum) a cinco herbicidas seletivos.

\section{MATERIAL E MÉTODOS}

O experimento foi instalado em casa de vegetação do Departamento de Produção Vegetal da Escola Superior de Agricultura "Luiz de Queiroz” da Universidade de São Paulo, município de Piracicaba-SP. De acordo com o Departamento de Ciências Exatas da USP/ ESALQ, neste município, a temperatura média do mês mais quente está acima de $24{ }^{\circ} \mathrm{C}$, e a do mês mais frio, abaixo de $17^{\circ} \mathrm{C}$.

O solo utilizado no experimento foi coletado de um pomar de citros que vinha sendo cultivado com a cultura por vários anos, mas sem a utilização de herbicidas residuais. Esta coleta foi feita com trado de $4,3 \mathrm{~cm}$ de diâmetro, de 0 a $10 \mathrm{~cm}$ de profundidade (o volume de solo coletado foi o necessário para encher os vasos). Depois disso, este solo foi colocado em sacos plásticos, levado ao laboratório, peneirado (peneira com malha de $0,280 \mathrm{~mm}$ ), para desfazer possiveis agregados, e colocado em vasos plásticos de dois litros de capacidade $(10 \mathrm{~cm}$ de diâmetro superior, $7 \mathrm{~cm}$ de diâmetro inferior e $10 \mathrm{~cm}$ de altura). A análise física do solo revelou $43 \%$ de areia total, $19 \%$ de silte e $38 \%$ de argila, sendo ele classificado como de textura argilosa, com pH de 5,3 e $37 \mathrm{~g} \mathrm{dm}^{-3}$ de matéria orgânica.

As mudas das espécies de grama estudadas foram produzidas em tubetes, que foram transplantadas nos vasos, sendo colocados quatro tubetes por vaso. O sistema de irrigação da casa de vegetação era composto por microaspersores, que eram controlados por um "timer", irrigando aproximadamente $5 \mathrm{~mm}$ a cada acionamento, três vezes ao dia. A casa de vegetação possuía um sistema automático de controle de temperatura e umidade relativa, por meio de ventiladores, sendo a luminosidade controlada por uma cobertura de sombrite, que era, automaticamente, distendida de acordo com a luminosidade incidente. 
O ensaio foi instalado em duas etapas, ou seja, os tratamentos herbicidas pré-plantio (sulfentrazone a $1,4 \mathrm{~kg}$ i.a ha ${ }^{-1}$ e oxadiazon a $1,0 \mathrm{~kg}$ i.a ha-1) foram aplicados antes do plantio das variedades de grama (15 de janeiro de 2000), e os herbicidas aplicados em pós-emergência (halosulfuron a 112,5 g i.a ha ${ }^{-1}, 2,4-\mathrm{D}$ a 2.010 g i.a ha ${ }^{-1}$ e MSMA a 2,4 kg i.a ha-1), 15 dias após o transplante dos tubetes de grama, além de uma testemunha em que não foi feita a aplicação de herbicidas. A aplicação de herbicidas foi feita em câmara de pulverização especialmente construída para aplicação de herbicidas em vasos, utilizando ponta de pulverização do tipo $8002 \mathrm{E}$, com pressão de trabalho de $3 \mathrm{kPa}$ e consumo de calda de $200 \mathrm{~L} \mathrm{ha}^{-1}$. Todos os tratamentos herbicidas foram aplicados nas variedades de grama são-carlos, esmeralda, bermuda, zoysia e santo-agostinho. O delineamento experimental adotado foi o completamente casualizado, com quatro repetições, em esquema fatorial 5x5 mais a testemunha, ou seja, cinco tratamentos herbicidas e cinco espécies de grama, além da testemunha.

As avaliações foram feitas visualmente, adotando-se uma escala proposta pela EWRC (European Weed Research Council), que varia de um a nove, em que: 1 - ausência de sintomas de toxicidade; 2 - sintomas de toxicidade muito leves; 3 - sintomas de toxicidade leves; 4 - sintomas de toxicidade considerados moderados; 5 - sintomas classificados como duvidosos, em que o avaliador não tem certeza se eles causarão toxicidade ao gramado; 6 - sintomas que aparentam causar toxicidade leve; 7 - com toxidade forte; 8 - com toxicidade muito forte; e 9 - morte das plantas. Os resultados foram analisados estatisticamente por meio de análise de variância, para constatar a significância estatística dos tratamentos. Em seguida, foi feita regressão polinomial para ajuste de equações matemáticas.

\section{RESULTADOS E DISCUSSÃO}

Os resultados da avaliação dos sintomas de toxicidade nas cinco espécies de grama estão representados nas Figuras 1 a 5, por meio da regressão quadrática dos dados. A grama bermuda sofreu injúria severa, principalmente pelos herbicidas oxadiazon e sulfentrazone, ou seja, aqueles aplicados em condições de pré-plantio da grama (Figura 1). Aos efeitos fitotóxicos destes herbicidas foram atribuídas notas com média em torno de 7 nas últimas avaliações, efetuadas aos 50 dias após a aplicação dos herbicidas, não mostrando nenhum sinal de recuperação. Já o MSMA foi menos tóxico para a grama bermuda, porém os valores de toxicidade ainda não foram aceitáveis na prática. O herbicida que proporcionou a menor toxicidade para a grama bermuda foi o halosulfuron, uma vez que os índices máximos de toxicidade atingidos ficaram em torno de 2,5, aos 40 dias após a aplicação do herbicida, com recuperação evidente a partir dos 50 dias após aplicação (Figura 1). Os sintomas de injúrias causados pelo 2,4-D também foram bastante aceitáveis, sendo levemente superiores aos do halosulfuron, porém aos 50 dias após a aplicação do herbicida estes sintomas foram iguais para os dois herbicidas. Dessa forma, conclui-se que, para a variedade de grama bermuda, os herbicidas que podem ser aplicados nas doses estudadas nesta pesquisa, com níveis de toxicidade aceitáveis, são o halosulfuron a $112,5 \mathrm{~g}$ i.a ha ${ }^{-1}$ e o 2,4-D a $2.010 \mathrm{~g}$ i. a ha-1. Os índices visuais de toxicidade causados pelo sulfentrazone a $1,4 \mathrm{~kg}$ i.a ha-1, pelo oxadiazon a $1,0 \mathrm{~kg}$ i.a ha ${ }^{-1}$ e pelo MSMA a $2,4 \mathrm{~kg}$ i.a ha-1 não são aceitáveis na prática, comprometendo de forma significativa o desenvolvimento e a aparência visual da grama bermuda (Figura 1).

Foloni e Camposilvan (1997) estudaram o controle da planta daninha tiririca (Cyperus rotundus) em gramados. Para isso, foi aplicado o herbicida halosulfuron nas doses de 75 a 225 g i.a ha-1, em comparação com o 2,4-D na dose de 1,34 kg i.a ha-1, nas variedades de grama batatais, esmeralda, zoysia, santo-agostinho e são-carlos. Os autores concluíram que o halosulfuron é uma alternativa no controle da tiririca em áreas de gramado, sem, entretanto, afetar severamente o desenvolvimento e a aparência da grama.

Os resultados na grama santo-agostinho (Figura 2) foram bastante parecidos com o descrito para a grama bermuda. No entanto, os índices de toxicidade observados para os herbicidas halosulfuron a $112,5 \mathrm{~g}$ i.a ha $\mathrm{ha}^{-1}$ e ,4-D a $2.010 \mathrm{~g} \mathrm{i}^{\mathrm{a} \mathrm{ha}} \mathrm{h}^{-1}$ foram maiores que os observados para a bermuda, inclusive não mostrando sinais de recuperação da toxicidade, mesmo 
aos 50 dias após a aplicação dos herbicidas, com notas em torno de 3 a 4 (Figura 2). Os niveis de toxicidade para os herbicidas sulfentrazone a $1,4 \mathrm{~kg}$ i.a ha ${ }^{-1}$, MSMA a $2,4 \mathrm{~kg}$ i.a ha-1 e oxadiazon a $1,0 \mathrm{~kg}$ i.a ha-1 foram semelhantes, conforme pode ser observado nas curvas de regressão quadrática apresentadas na Figura 2. Dessa forma, no caso de uma recomendação mais segura de herbicida para a grama santo-agostinho, é necessário que novas pesquisas sejam desenvolvidas, principalmente no campo, para observação dos reais danos causados pelos herbicidas no desenvolvimento vegetativo da grama santoagostinho.

Os resultados para a grama zoysia foram difíceis de ser interpretados, como pode ser observado na Figura 3. No entanto, foi uma grama que se mostrou bastante tolerante aos herbicidas, apesar de, graficamente, parecer que os sintomas tenham sido severos; é importante salientar que a escala de toxicidade colocada no gráfico varia de 0 a 4 (Figura 3), e

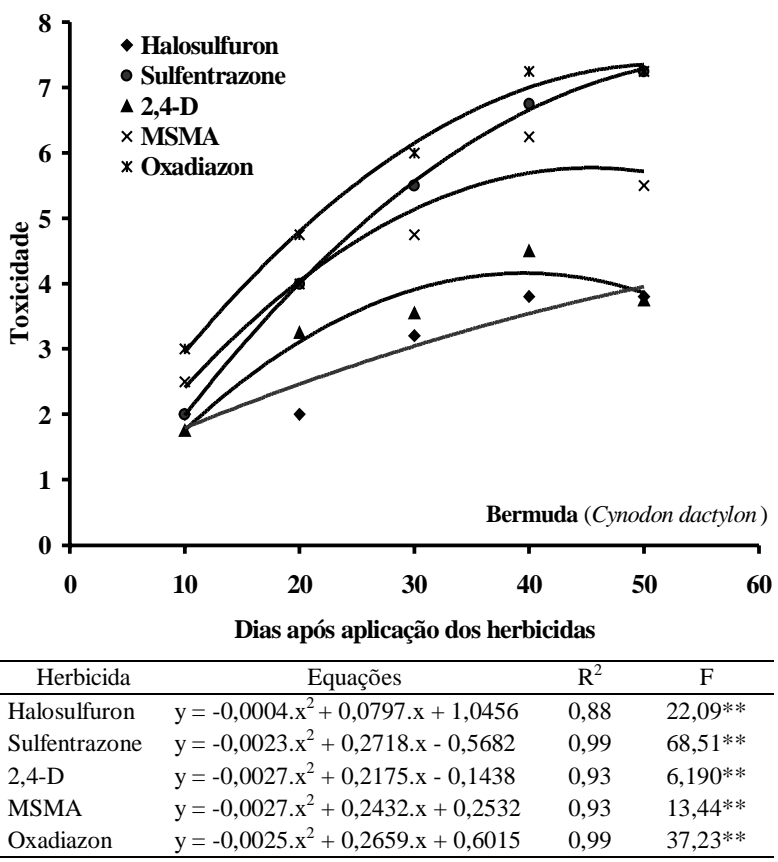

Figura 1 - Avaliação da toxicidade causada pelos herbicidas na grama bermuda (Cynodon dactylon); parâmetros das equações de regressão, valores dos coeficientes de correlação e valores de $\mathrm{F}$ da análise de variância $(* *=$ significativo estatisticamente a $5 \%$ de probabilidade pelo teste F). Piracicaba-SP, 2000. nos gráficos anteriores as escalas variaram de 0 a 8 (Figura 1) e 0 a 9 (Figura 2). Portanto, todos os herbicidas aplicados provocaram injúrias consideradas leves e aceitáveis, na prática, para esta variedade de grama. A maior recuperação da grama zoysia aos sintomas iniciais de toxicidade foi observada com o herbicida oxadiazon a $1,0 \mathrm{~kg}$ i.a ha-1, cujos índices chegaram a 1,0 em média (Figura 3). Os demais herbicidas estudados também tiveram índices finais de toxicidade considerados aceitáveis. Assim, pode-se concluir que a grama zoysia apresenta elevado grau de tolerância aos herbicidas estudados, e todos eles poderiam ser aplicados em gramados desta espécie, com injúrias consideradas leves.

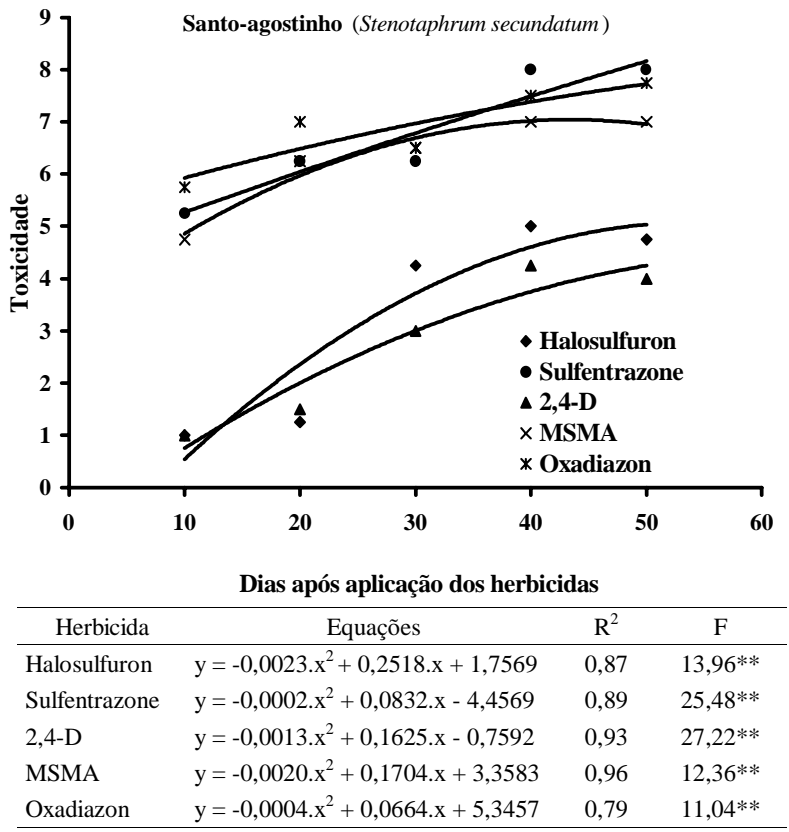

Figura 2 - Avaliação da toxicidade causada pelos herbicidas na grama santo-agostinho (Stenotaphrum secundatum): parâmetros das equações de regressão, valores dos coeficientes de correlação e valores de $\mathrm{F}$ da análise de variância $(* *=$ significativo estatisticamente a $5 \%$ de probabilidade pelo teste F). Piracicaba-SP, 2000.

Johnson \& Carrow (1999) estudaram a seletividade de herbicidas pré-emergentes em oito cultivares de gramas do tipo zoysia. O oxadiazon foi o único herbicida que não reduziu significativamente a qualidade de qualquer dos cultivares estudados de zoysia. A tolerância foi intermediária ao pendimethalin; já oryzalin e 
dithiopyr causaram alta toxicidade, não sendo, portanto, recomendados. Segundo os resultados obtidos por estes autores, a tolerância a herbicidas pela zoysia, além de ser dependente da variedade, é função também da dose e época do ano em que o herbicida é aplicado.

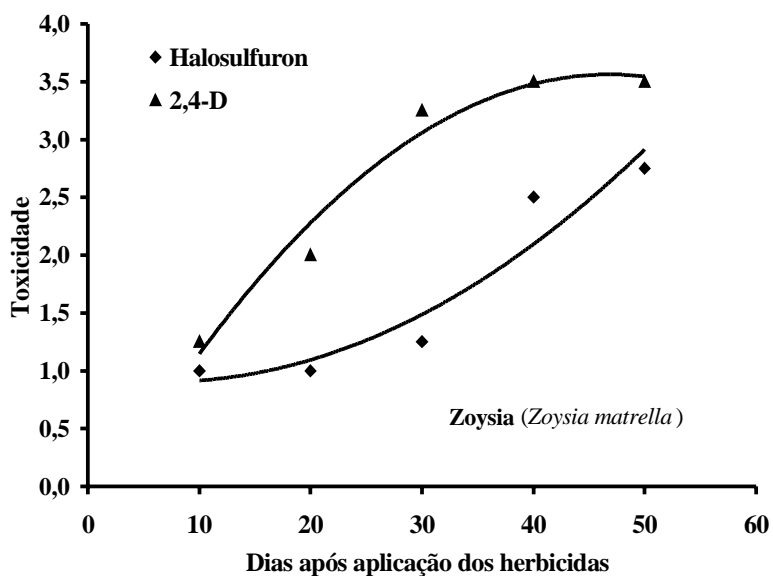

\begin{tabular}{llcc}
\hline \multicolumn{1}{c}{ Herbicida } & \multicolumn{1}{c}{ Equações } & $\mathrm{R}^{2}$ & $\mathrm{~F}$ \\
\hline Halosulfuron & $\mathrm{y}=0,0011 \cdot \mathrm{x}^{2} 0,0143 \cdot \mathrm{x}-0,0143$ & 0,90 & $17,64^{* *}$ \\
Sulfentrazone & média $=1,75$ & --- & $3,06 \mathrm{n} . \mathrm{s}$. \\
$2,4-\mathrm{D}$ & $\mathrm{y}=-0,0018 \cdot \mathrm{x}^{2}+0,1671 . \mathrm{x}-0,3575$ & 0,96 & $18,78^{* *}$ \\
MSMA & média $=1,85$ & --- & $5,00 \mathrm{n} . \mathrm{s}$. \\
Oxadiazon & média $=1,25$ & --- & $3,37 \mathrm{n} . \mathrm{s}$. \\
\hline
\end{tabular}

Figura 3 - Avaliação da toxicidade causada pelos herbicidas na grama zoysia (Zoysia matrella); parâmetros das equações de regressão, valores dos coeficientes de correlação e valores de $\mathrm{F}$ da análise de variância $(* *=$ significativo estatisticamente a $5 \%$ de probabilidade pelo teste $\mathrm{F}$; n.s. = não-significativo, portanto não houve variabilidade nos dados). Piracicaba-SP, 2000.

Na Figura 4 estão representados os resultados da variedade de grama esmeralda; como era esperado, os níveis de tolerância desta grama aos herbicidas é grande (Figura 4), comparado com a zoysia (Figura 3), pois ambas pertencem ao mesmo gênero botânico, ou seja, grama Esmeralda (Zoysia japonica) e Zoysia (Zoysia matrella). Os herbicidas de maior tolerância pela grama esmeralda são MSMA a $2,4 \mathrm{~kg}$ i.a ha-1, 2,4-D a 2.010 g i.a ha-1 e halosulfuron a $112,5 \mathrm{~g}$ i.a ha-1 (Figura 4). Os herbicidas oxadiazon a $1,0 \mathrm{~kg}$ i.a ha ${ }^{-1}$ e sulfentrazone a 1,4 kg i.a ha-1 foram os de menor seletividade para a grama esmeralda, porém em niveis aceitáveis de toxicidade na prática. Uma das prováveis razões da seletividade dos herbicidas pós-emergentes às gramas do gênero Zoysia é decorrente do formato das folhas, que é normalmente estreito e curto, quando comparado com as demais variedades de grama; e esta característica dificulta a retenção do herbicida na superfície da folha.

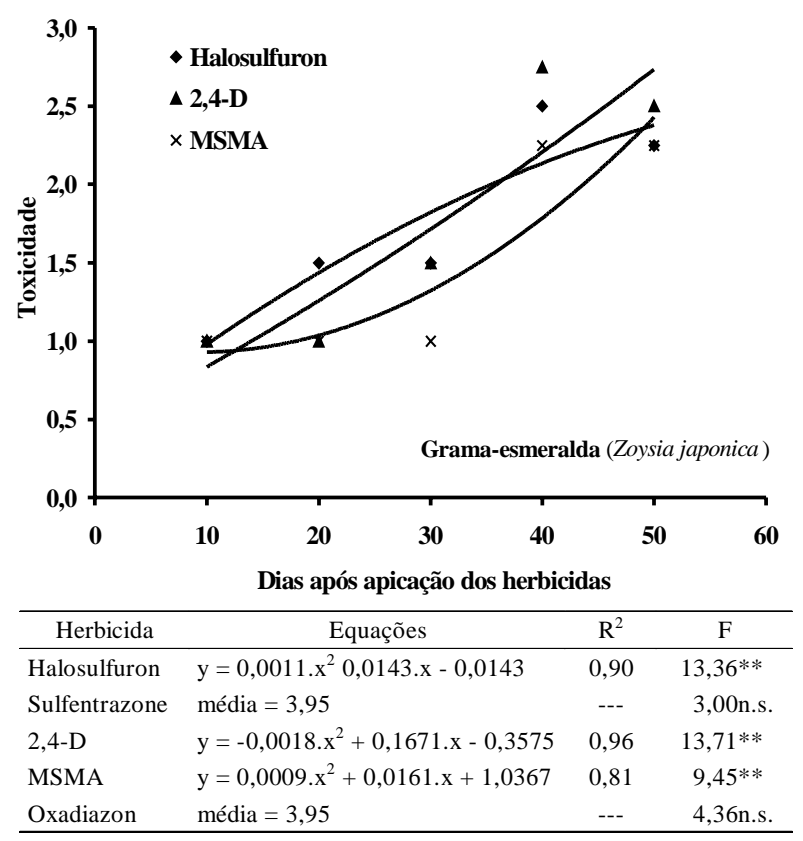

Figura 4 - Avaliação da toxicidade causada pelos herbicidas na grama esmeralda (Zoysia japonica): parâmetros das equações de regressão, valores dos coeficientes de correlação e valores de $\mathrm{F}$ da análise de variância $(* *=$ significativo estatisticamente a $5 \%$ de probabilidade pelo teste F; n.s. = não-significativo, portanto não houve variabilidade nos dados). Piracicaba-SP, 2000.

Carmona \& Silva (1997) testaram a seletividade de herbicidas à grama esmeralda e concluíram que atrazine, atrazine + simazine, atrazine + óleo vegetal, asulan e flazasulfuron não apresentaram efeito fitotóxico quando aplicados em doses normalmente recomendadas para culturas. Segundo estes autores, os herbicidas ametryne, imazapyr e triclopyr, nas doses estudadas na pesquisa, não devem ser recomendados para a grama esmeralda, pois causaram-lhe sintomas de danos de pelo menos $10 \%$.

A seletividade do herbicida sulfentrazone na dose de 1,4 kg i.a ha-1 para a grama sãocarlos é quase que total (Figura 5), resultado este contrário aos observados nas demais 
variedades de grama estudadas nesta pesquisa. As médias dos sintomas visuais de toxicidade causados pelo sulfentrazone foram próximas de 1, comprovando ser um herbicida seletivo para esta grama. Contrariamente, o MSMA a $2,4 \mathrm{~kg}$ i.a ha-1 não foi seletivo, chegando a causar morte de plantas de grama são-carlos. Sinais de toxicidade foram observados em torno dos 30 dias após a aplicação de oxadiazon a $1,0 \mathrm{~kg}$ i.a ha ${ }^{-1}$, porém estes sintomas desapareceram aos 50 dias após a aplicação do herbicida (Figura 5). Halosulfuron a 112,5 g i.a ha ${ }^{-1} \mathrm{e}$ 2,4-D a $2.010 \mathrm{~g}^{\mathrm{i}}$ a $\mathrm{ha}^{-1}$ tiveram seletividade intermediária, mas aceitáveis do ponto de vista prático.

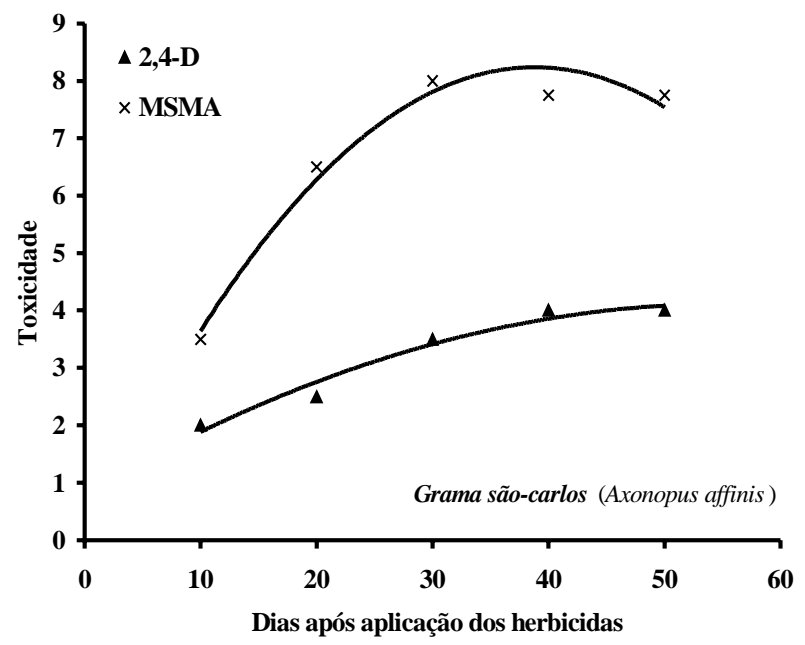

\begin{tabular}{llcc}
\hline \multicolumn{1}{c}{ Herbicida } & \multicolumn{1}{c}{ Equações } & $\mathrm{R}^{2}$ & $\mathrm{~F}$ \\
\hline Halosulfuron & média $=3,6$ & --- & $3,56 \mathrm{n} . \mathrm{s}$. \\
Sulfentrazone & média $=1,65$ & --- & $0,45 \mathrm{n} . \mathrm{s}$. \\
$2,4-\mathrm{D}$ & $\mathrm{y}=-0,0018 \cdot \mathrm{x}^{2}+0,1671 . \mathrm{x}-0,3575$ & 0,96 & $33,00^{* *}$ \\
MSMA & $\mathrm{y}=-0,0055 \cdot \mathrm{x}^{2}+0,4296 . \mathrm{x}-0,1000$ & 0,97 & $6,11^{*}$ \\
Oxadiazon & média $=2,95$ & --- & $0,18 \mathrm{n} . \mathrm{s}$. \\
\hline
\end{tabular}

Figura 5 - Avaliação da toxicidade causada pelos herbicidas na grama são-carlos (Axonopus affinis); parâmetros das equações de regressão, valores dos coeficientes de correlação e valores de $\mathrm{F}$ da análise de variância $(* *=$ significativo estatisticamente a $5 \%$ de probabilidade pelo teste F; n.s. não-significativo, portanto não houve variabilidade nos dados). Piracicaba-SP, 2000.
A conclusão principal desta pesquisa é de que a seletividade de herbicidas aos diversos tipos de grama é dependente da variedade de grama e do tipo de herbicida utilizado. Não existe uma regra única de recomendação de herbicidas, sendo, portanto, necessárias recomendações diferenciadas, dependendo do nível de toxicidade tolerado. Este trabalho de pesquisa fornece informações de forma científica sobre a seletividade de herbicidas para gramados; no entanto, novas pesquisas são necessárias para quantificar melhor a toxicidade dos herbicidas no campo, além da determinação de fatores edáficos e climáticos na seletividade de herbicidas para gramados.

\section{LITERATURA CITADA}

BHOWMIK, P.C.; NEAL, J.C. Turfgrass weed control strategies. In: ANNUAL MEETING OF THE NORTHEASTERN WEED SCIENCE SOCIETY, 49, 1995, Raleigh. Proceedings... Raleigh: NWSC, 1995. p.154-155.

CARMONA, R.; SILVA, F.X. Seletividade da grama esmeralda (Zoysia japonica) a herbicidas. In: CONGRESSO BRASILEIRO DA CIÊNCIA DAS PLANTAS DANINHAS, 21, 1997, Caxambu. Resumos... Caxambu: SBCPD, 1997. p.324.

FOLONI, L.L.; CAMPOSILVAM, D. Seletividade de halosulfuron em cinco diferentes tipos de gramados. In: CONGRESSO BRASILEIRO DA CIÊNCIA DAS PLANTAS DANINHAS, 21, 1997, Caxambu. Resumos... Caxambu: SBCPD, 1997. p.326.

FREITAS, F.C.; FREITAS, L.H.L.; FERREIRA, L.R., GONSALVES, W. Influência de cimetacarb e clethodim como reguladores de crescimento em gramado. In: CONGRESSO BRASILEIRO DA CIÊNCIA DAS PLANTAS DANINHAS, 21, 1997, Caxambu: Resumos... Caxambu: SBCP, 1997. p.325.

ITOGRASS. Gramados. São Paulo, Europa, 1997. $67 \mathrm{p}$.

JOHNSON, B.J.; CARROW, R.N. Tolerance of Zoysiagrass (Zoysia spp.) cultivars to preemergence herbicides. Weed Technol., v.13, n.4, p.706-712, 1999. 